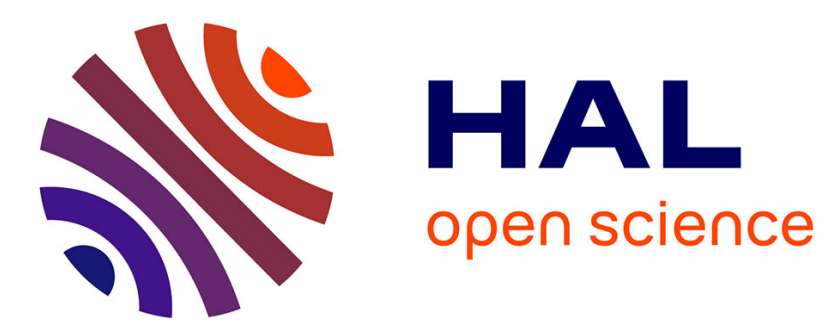

\title{
Experimental multiphoton ionization of cesium atoms through the 9D $3 / 2$ resonant level
}

\author{
G. Brincourt
}

\section{To cite this version:}

G. Brincourt. Experimental multiphoton ionization of cesium atoms through the 9D 3/2 resonant level. Journal de Physique Lettres, 1977, 38 (3), pp.81-84. 10.1051/jphyslet:0197700380308100 . jpa-00231331

\section{HAL Id: jpa-00231331 https://hal.science/jpa-00231331}

Submitted on 1 Jan 1977

HAL is a multi-disciplinary open access archive for the deposit and dissemination of scientific research documents, whether they are published or not. The documents may come from teaching and research institutions in France or abroad, or from public or private research centers.
L'archive ouverte pluridisciplinaire HAL, est destinée au dépôt et à la diffusion de documents scientifiques de niveau recherche, publiés ou non, émanant des établissements d'enseignement et de recherche français ou étrangers, des laboratoires publics ou privés. 


\title{
Classification \\ Physics Abstracts 5.270 \\ EXPERIMENTAL MULTIPHOTON IONIZATION OF CESIUM ATOMS THROUGH THE 9D 9 $_{3 / 2}$ RESONANT LEVEL
}

\author{
G. BRINCOURT (*) \\ Université de Provence, Centre de St-Jérôme, 13397 Marseille Cedex 4, France
}

(Reçu le 20 septembre 1976, révisé le 3 décembre 1976, accepté le 22 décembre 1976)

\begin{abstract}
Résumé. - Nous présentons dans cette lettre une expérience d'ionisation multiphotonique d'atomes de césium à partir du niveau résonnant $9 \mathrm{D}_{3 / 2}$, à l'aide d'un laser à rubis. La courbe de dispersion de la résonance est comparée à l'allure de la courbe théorique d'ionisation multiphotonique résonnante déduite des calculs de B. L. Beers et L. Armstrong [1] pour le césium.
\end{abstract}

\begin{abstract}
In this letter we report the results of an experiment concerning the multiphoton ionization of cesium atoms through the $9 \mathrm{D}_{3 / 2}$ resonant level, using a ruby laser. The experimental dispersion curve of the resonance obtained is compared to the theoretical shape of the resonant multiphoton ionization profile for cesium deduced from the calculations of B. L. Beers and L. Armstrong [1].
\end{abstract}

1. Introduction. - It has been shown $[1,2]$ that resonant multiphoton line shapes may be predicted in well defined experimental cases. Also, the possible asymmetry of some experimental line shapes may be explained [2] using a formalism introduced by Fano [3] to treat the autoionization problem.

In [1] an exact solution was obtained for a realistic model for two-photon ionization, and applied to two and three-photon ionization in cesium. In this latter case the ionization is performed via the $9 D_{3 / 2,5 / 2}$ states. As a matter of fact, for cesium atoms the $6 \mathrm{~S}_{1 / 2}-9 \mathrm{D}_{3 / 2}$ transition $\left(28828.90 \mathrm{~cm}^{-1}\right)$, is nearly resonant with the two-photon energy $\left(28802 \mathrm{~cm}^{-1}\right)$ of a ruby laser at room temperature $(\lambda \simeq 6942 \AA$ in air). By cooling the ruby rod it is possible to reach and also exceed the $9 D_{3 / 2}$ level with two photons.

Previous observations of two-photon absorption on this level in cesium were first made by Abella [4]. Ionization via this 9D state was then performed by several authors [5-8]. Also ionization of cesium via the $6 \mathrm{~F}$ level was investigated using a neodymiumglass laser $[9,10]$.

2. Theoretical description of the phenomenon. The theoretical treatment used in [1] is carried out with the following simplifying assumptions connected with the experiments : the laser pulse shape is supposed to be rectangular, and the photons are assumed to be of the same frequency in a single mode and linearly

$\left(^{*}\right)$ Work conducted in the Laboratoire de Photoélectricité, Université d'Aix-Marseille III, St-Jérôme, 13397 Marseille Cedex 4. polarized. The resonant $9 \mathrm{D}_{3 / 2,5 / 2}$ states of cesium are supposed to be degenerate, and the groundstate hyperfine splitting of $\simeq 0.3 \mathrm{~cm}^{-1}[11]$, [8] is not considered. One can find in detail in [1] other simplifying assumptions used for the calculation.

The theoretical results may be summarized as follows : in the case of ionization the number of ions formed is given by $N_{\mathrm{i}}=N_{0} P(T)$ where $N_{0}$ is the number of atoms in the interaction region, $T$ the length of the laser pulse and $P(T)$ the overall probability for an atom to be ionized. From our experimental data (presented later) $P(T)$ must be approximated (see [1]) by :

with

$$
P_{2}(T)=1-\mathrm{e}^{-\gamma_{2} T}
$$

when

$$
\gamma_{2}=\frac{\gamma_{\mathrm{g}}}{2}\left(1+\frac{\left(\delta+\gamma_{\mathrm{a}} q\right)^{2}}{\delta^{2}+4\left|H_{\mathrm{ga}}\right|^{2}}\right)
$$

$$
2\left|H_{\mathrm{ga}}\right| \gg \frac{1}{2} \gamma_{\mathrm{a}}
$$

(the index 2 refers to Equations (17) and (18) in [1]).

$\delta$ is the dynamic detuning which here is the difference between the energy of two photons $2 \hbar \omega$, and the energy of the $6 S_{1 / 2} \rightarrow 9 D_{3 / 2}$ transition for which bound state energies of the ground state $|6 \mathrm{~S}\rangle$ and the resonant state $\mid$ 9D $\rangle$ are supposed shifted principally by their interaction with the continuum :

$$
\delta=\delta_{0}+\delta E_{6 \mathrm{~S} \rightarrow 9 \mathrm{D}}
$$


where

$$
\delta_{0}=\left(E_{6 \mathrm{~S}}-E_{9 \mathrm{D}}\right)+2 \hbar \omega
$$

and

$$
\delta E_{6 \mathrm{~S} \rightarrow 9 \mathrm{D}}=\Delta E_{6 \mathrm{~S}}-\Delta E_{9 \mathrm{D}}
$$

$\delta_{0}$ is the field-free (or static) detuning and $\delta E_{6 \mathrm{~S} \rightarrow 9 \mathrm{D}}$ the energy shift of the transition. At exact dynamic resonance $\delta=0$.

$\gamma_{\mathrm{g}}$ and $\gamma_{\mathrm{a}}$ are the ionization decay widths of the $|\mathrm{g}\rangle$ and $|\mathrm{a}\rangle$ states, which are here respectively the $|6 \mathrm{~S}\rangle$ and $\mid$ 9D $\rangle$ states.

$q$ is a parameter measuring the interference between the resonant and nonresonant processes and determines the shape of the resonant multiphoton ionization profiles. This parameter is identical with that introduced by Fano [3] in the study of autoionization processes and is related to $H_{\mathrm{ga}}, \gamma_{\mathrm{a}}$ and $\gamma_{\mathrm{g}}$ by the relation :

$$
q=\frac{2 H_{\mathrm{ga}}}{\left|\gamma_{\mathrm{a}} \gamma_{\mathrm{g}}\right|^{1 / 2}}
$$

Also $\gamma_{\mathrm{a}}, \gamma_{\mathrm{g}}$ and $H_{\mathrm{ga}}$ depend on the atomic species. For cesium their expressions versus laser intensity are given in [1] assuming linear polarization of the light, and for a single mode frequency corresponding to $\lambda \simeq 6920 \AA$. One can note that eq. (1), (2) lead to an asymmetric dispersion curve in $\delta$, the other parameters being held fixed.

But in the three-photon ionization case for cesium and with the laser intensity considered in our experiments $\left(I \simeq 10^{7} \mathrm{~W} / \mathrm{cm}^{2}\right)$, we have $\gamma_{\mathrm{a}} q \gg \delta$ in the vicinity of the resonance. Consequently no asymmetry may appear, and eq. (2) then can be simplified :

$$
\gamma_{2} \simeq \frac{\gamma_{\mathrm{g}}}{2} \frac{\gamma_{\mathrm{a}}^{2} q^{2}}{\delta^{2}+4\left|H_{\mathrm{ga}}\right|^{2}} .
$$

3. Experimental investigation. - The experiment is performed as follows : we focus linear polarized light from a rotating prism $Q$-switched ruby laser onto a perpendicular atomic cesium beam flowing from a tank at room temperature in ultra high vacuum. The tank at its top has a small rectangular slit $1 \mathrm{~cm}$ long and $0.05 \mathrm{~cm}$ wide followed by a collimator of the same aperture. The cesium vapour pressure at $20^{\circ} \mathrm{C}$ is $\simeq 10^{-6}$ torr [12] and simultaneously the pressure in the vacuum group is $\simeq 3 \times 10^{-8}$ torr. A cesium beam thus flows continuously from the tank. At such a low temperature no $\mathrm{C}_{\mathrm{S}_{2}}$ component perturbation is present as verified. The density of cesium atoms in the interaction volume is $\simeq 1.6 \times 10^{7} \mathrm{At} / \mathrm{cm}^{3}$ and is determined by a hotwire detector. The diaphragmed laser beam is $0.6 \mathrm{~cm}$ in diameter and is focused by a lens of focal length $33 \mathrm{~cm}$ in the atomic cesium beam and perpendicular to the motion of the atoms to greatly reduce Doppler effects. Under such a weak focusing the interaction volume is essentially determined by the width of the cesium beam $(0.05 \mathrm{~cm})$ in the direction of light propagation, and transversely by the section of the laser beam in the focal section. The created ions are accelerated by a d.c. electric field of $1500 \mathrm{~V} / \mathrm{cm}$ and are detected then identified by a time of flight mass spectrometer including an electron multiplier (type 50 P3R-R.T.C.) with an over-all gain of $3 \times 10^{4}$.

The ion current is measured using a high-gain amplifier and an oscilloscope, whose bandwidths have been taken into account in the determination of the number of ions $N_{\mathrm{i}}$. A fast photocell and a calorimeter measure the output laser power and energy. The photocell output is recorded on a $500 \mathrm{MHz}$ bandwidth oscilloscope. The mode structure of the laser is observed with a Fabry-Pérot interferometer with $100 \mathrm{MHz}$ resolution.

The laser frequency is tuned by cooling the ruby rod with gaseous nitrogen passed through liquid nitrogen. The temperature is determined by a calibrated thermocouple fixed on the rod and is adjusted by varying the gas flow. The wavelength of the laser is calculated from the position of the laser line relative to the neon lines on photographic plates of a grating spectrograph with an average dispersion of $1.9 \AA / \mathrm{mm}$ and with a resolution of $2 \mathrm{GHz}\left(\simeq 0.07 \mathrm{~cm}^{-1}\right)$. An output quadratic dispersion law $\lambda(x)$ has been considered. Absolute calibration curve $\sigma(T)$ of the wave number of the laser light versus temperature in the range considered is given figure 1 .

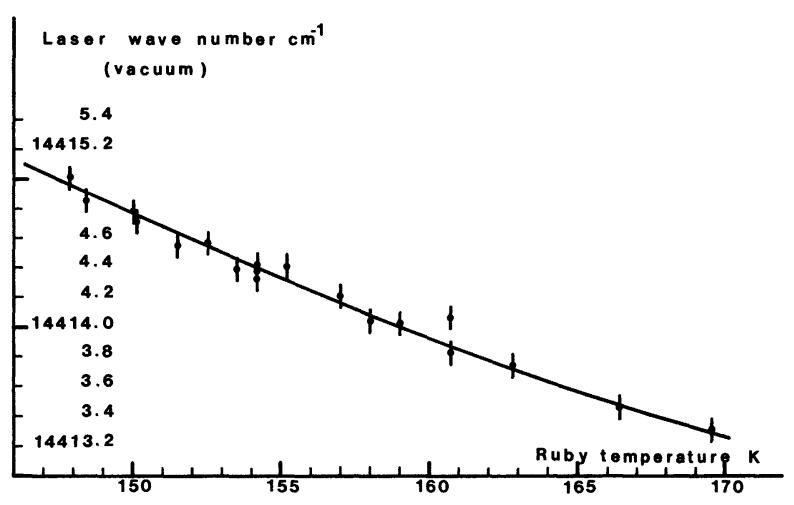

FIG. 1. - Calibration curve of the temperature dependence of the ruby laser emission. Each point refers to a single shot. The vertical error bars concern the calculated uncertainly of the laser wave number.

The theoretical value of $\sigma$, in vacuum, corresponding exactly to two-photon absorption on the $9 D_{3 / 2}$ cesium level is $28828.90 \mathrm{~cm}^{-1}$ from Moore's tables [13]. From our calibration curve this corresponds approximately to a temperature of $153.5 \mathrm{~K}$ for the ruby rod. In this range the slope is $\simeq 8 \times 10^{-2} \mathrm{~cm}^{-1} /$ degree.

The laser pulse length is $T \simeq 40 \mathrm{~ns}$ (full width at half maximum : FWHM) and the mean laser peak intensity is $\simeq 10^{7} \mathrm{~W} / \mathrm{cm}^{2}$ leading to a field $E \simeq 6 \times 10^{4} \mathrm{~V} / \mathrm{cm}$ in the interaction volume.

For each laser shot we record simultaneously the ion signal, the laser pulse shape, the energy, and the tem- 
perature of the ruby rod. The calibration curve $\sigma(T)$ is obtained separately, as are the results of the laser mode structure study.

Since the order of non-linearity $k$ (for cesium and off resonance $k=k_{0}=3$ ) of the multiphoton process generally depends on the laser frequency in the vicinity of a resonance [10], [15] no fully satisfying normalization or corrections to $N_{\mathrm{i}}$ are possible to rectify the non-reproductibility of the laser shots. Consequently, only results concerning pulses of practically constant peak power and shape were kept and reported.

4. Results and discussion. - One of the experimental dispersion curves obtained is shown in figure 2 (curve E) compared to that given by eq. (1), (5) (curve T). Each experimental point plotted corresponds to an individual laser shot. The vertical error bars concern the calculated uncertainties on $P(T)$ assuming an uncertainty of $20 \%$ in the interaction volume which is geometrically defined in paragraph 3

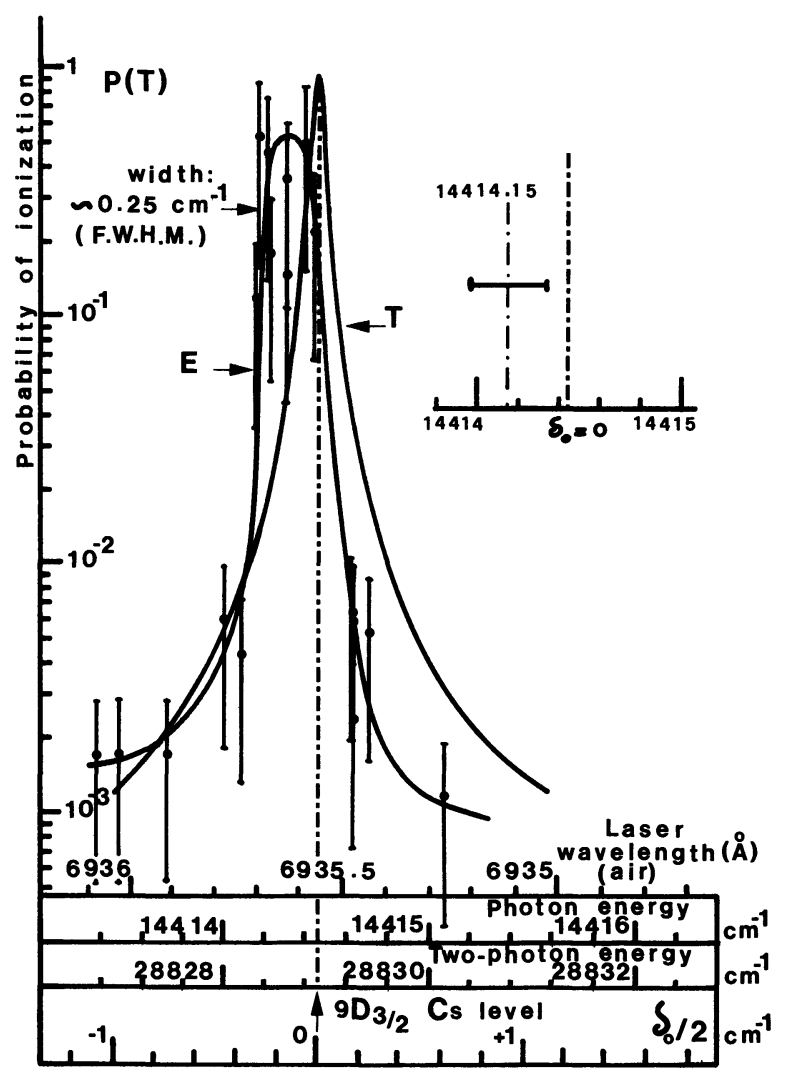

FIG. 2. - Experimental (E) and theoretical (T) dispersion curves for $P(T)$. On the $x$ axis are indicated the laser wavelength, the energy corresponding to one and two photons, and the energy difference $\delta_{0} / 2$ for both curves to be compared in width on the same one photon energy scale. The static position of the $9 \mathrm{D}_{3 / 2}$ resonant level is also given $\left(\delta_{0}=0\right)$. Curve (T) refers to eq. (1) (5) with the following values of the parameters corresponding to a laser intensity of $10^{7} \mathrm{~W} / \mathrm{cm}^{2}: \gamma_{\mathrm{g}} \simeq 3 \times 10^{-13} \mathrm{~cm}^{-1}$; $\gamma_{\mathrm{a}} \simeq 5 \times 10^{-3} \mathrm{~cm}^{-1} ; H_{\mathrm{ga}} \simeq 2 \times 10^{-2} \mathrm{~cm}^{-1} ; q \simeq 10^{6}$. The pulse length is $T \simeq 40 \mathrm{~ns}$. Each point corresponds to a single shot. The vertical error bars refer to the expected precision on the calculation of $P(T)$. The horizontal error bar quoted apart represents the uncertainty on the position of the maximum for four experimental curves : the mean experimental value is $14414.15 \mathrm{~cm}^{-1}$. but which depends in fact on the spatial distribution of the light in the focal zone and on the order of nonlinearity of the multiphoton process [16]. The horizontal error bar shown represents the position uncertainty of the maximum for four experimental dispersion curves. The mean value is found to be at $14414.15 \mathrm{~cm}^{-1}$. For simplicity all the linewidths and transition energies will be reported relative to one-photon energy, remembering that we have a two-photon resonant transition.

The general line shapes of both curves are roughly similar and we can make the following comments :

a) Under our experimental conditions and with low power near threshold pumping of the laser we have three or four principal longitudinal modes at room temperature, two modes below $180 \mathrm{~K}$, and often only one mode below $145 \mathrm{~K}$ using a $6 \mathrm{~cm}$ Fabry-Pérot interferometer for observations. The non-principal modes are estimated to have intensity lower than 2 to $3 \%$ of the principal modes. So we are almost in the same frequency conditions as were used for the theoretical calculations which were performed for a single mode frequency. In one mode operation the laser linewidth (FWHM) is found to be $\lesssim 0.02 \mathrm{~cm}^{-1}$. In two-mode operation the modes are separated by $0.05 \mathrm{~cm}^{-1}$, their width being $\$ 0.02 \mathrm{~cm}^{-1}$. Comparatively the calculated Doppler broadening for the transition considered due to the small divergence of the cesium beam is $\simeq 5 \times 10^{-4} \mathrm{~cm}^{-1}$ under our experimental conditions. Thus, disregarding the groundstate splitting, the width of the experimental dispersion curve is essentially governed by the laser linewidth.

The width (FWHM) of both curves $\mathrm{T}$ and $\mathrm{E}$ are respectively $\simeq 0.07 \mathrm{~cm}^{-1}$ and $\simeq 0.25 \mathrm{~cm}^{-1}$. Theoretically, for cesium and for the laser intensity used, the simplified eq. (1) is expected to overestimate the width by almost $30 \%$ compared to that predicted by the more complicated general equation (see [1]). So the experimental width is four or five times as wide as the theoretical one. The main reasons of such a discrepancy seem to be due to experimental uncertainties, and also to the presence of a ground-state hyperfine splitting which was not considered in the general theoretical calculation. Here the splitting is not resolved experimentally but it contributes to the width of the curve (about $50 \%$ at the top). Consequently we can note that the lower part of curve $T$ appears too wide.

b) The energy shift $\delta E_{6 \mathrm{~S} \rightarrow 9 \mathrm{D}}$ has not been evaluated theoretically. Curve $\mathrm{T}$ is then given versus the static detuning $\delta_{0}$ instead of the dynamic detuning $\delta$. The deviation is expected to be small as deduced from [10], where the energy shift of the $6 \mathrm{~S} \rightarrow 6 \mathrm{~F}$ transition in cesium using a neodymium glass laser is given for different values of the laser intensity $I$. One can deduce that $\delta E_{6 \mathrm{~S} \rightarrow 6 \mathrm{~F}} \simeq 2 \times 10^{-2} \mathrm{~cm}^{-1}$ for $I \simeq 10^{7} \mathrm{~W} / \mathrm{cm}^{2}$ in agreement with [9] and [14], after linear correction 
for the laser intensity. We can consider that we have the same order of value for $\delta E_{6 \mathrm{~S} \rightarrow 9 \mathrm{D}}$.

This value would not appreciably change the actual position of the theoretical curve $T$. Consequently the position of curve $E$ which is slightly shifted by about $0.2 \mathrm{~cm}^{-1}$ from the position of curve $\mathrm{T}\left(0.3 \mathrm{~cm}^{-1}\right.$ for the mean position of the obtained experimental curves) may be considered as good with respect to the theoretical prevision, the small shift being attributed to a possible systematic error.

c) The difference in magnitude of both curves may be attributed to several causes. First, to the incomplete knowledge of the effective interaction volume which is generally less than the geometrical one [16] which has been considered here. Second, to the uncertainty in the absolute value of the laser intensity (assuming an imprecision of $50 \%$ in the laser intensity leads to a change of $\simeq 20 \%$ in the value of $P(T)$ at $\delta_{0}=0$ ). Third, to the theoretical value of $P(T)$ which seems to be overestimated as expected in [1], since the experiments are generally situated in a transition region for cesium were the quantities $2\left|H_{\mathrm{ga}}\right|$ and $\frac{1}{2} \gamma_{\mathrm{a}}$ differ only slightly in value instead of the required condition $2\left|H_{\mathrm{ga}}\right| \gg \frac{1}{2} \gamma_{\mathrm{a}}$ (see eq. (3)).

In addition, a remark is to be made concerning the laser pulse shape. In [1] the pulse is supposed to be rectangular while experimentally it looks rather triangular. For the normalization used here we have attributed to the rectangular pulse the length deduced from the experimental pulse at half maximum (that is here $T \simeq 40 \mathrm{~ns}$ ). This normalization is all the less satisfying as the order of the non linear process $k$ is high, and would be acceptable only for a linear process that is for $k=1[15,16]$. And, roughly, for a given experimental laser pulse shape, the higher the value of $k$, the shorter would be the corresponding rectangular pulse in [1]. In consequence the actual normalization, which can not be corrected practically through a resonance, certainly leads to a greater ionization probability for the theoretical behaviour [17].

Another indication shows that the contribution of the $9 D_{5 / 2}$ level $\left(7 \mathrm{~cm}^{-1}\right.$ above the $9 D_{3 / 2}$ level $)$ to the ionization is negligible. This check is performed using the frequency dependant non-linear susceptibilities for both transitions calculated in [7]. We find that at $14415 \mathrm{~cm}^{-1}$, that is for the lower part of curve $\mathrm{E}$ towards the $9 \mathrm{D}_{5 / 2}$ level, the contribution of this level is $<5 \%$. Then no interference is to be considered due to the presence of the nearest $9 \mathrm{D}_{5 / 2}$ level.

5. Conclusion. - We have investigated the resonant three-photon ionization of cesium atoms through the $9 D_{3 / 2}$ level using an ion collection technique. The results are compared with the predictions of a theoretical paper. The comparison shows that some deviations observed may be attributed in part to experimental uncertainties, and in part to theoretical assumptions which could not all be experimentally satisfied (particularly the dropping of any groundstate splitting, and the consideration of a rectangular laser pulse shape this being, up to now, practically inconsistent with most of the experiments). Nevertheless the deviations are not severe and both theoretical and experimental improvements should provide more precise and fruitful comparisons.

Acknowledgments. - The author wishes to thank Dr. C. Manus, Dr. G. Mainfray and Dr. J. Morellec for many critical and helpful discussions, and Dr. Y. Gontier for his theoretical aid (C.E.A. Saclay).

\section{References}

[1] Beers, B. L. and Armstrong, L. Jr., Phys. Rev. A 12 (1975) 2447.

[2] Armstrong, L. Jr., Beers, B. L., Fenneuille, S., Phys. Rev. A 12 (1975) 1903.

Feneuille, S., Armstrong, L. Jr., J. Physique Lett. 36 (1975) L-235.

[3] Fano, U., Phys. Rev. 124 (1961) 1866.

[4] Abella, I. D., Phys. Rev. Lett. 9 (1962) 453.

[5] Evans, R. G. and Thonemann, P. C., Phys. Lett. 39A (1972) 133 and Phil. Mag. 28 (1973) 1387.

[6] Popescu, D., Collins, C. B., Johnson, B. W., Popescu, I., Phys. Rev. A 9 (1974) 1182.

[7] Leung, K. M., Ward, J. F., OrR, B. J., Phys. Rev. A 9 (1974) 2440.

[8] Ward, J. F., Smith, A. V., Phys. Rev. Lett. 35 (1975) 653.

[9] Held, B., Mainfray, G., Manus, C., Morellec, J., SanCHEZ, F., Phys. Rev. Lett. 30 (1973) 423.
[10] Morellec, J., Normand, D., Petite, G., Phys. Rev. A 14 (1976) 300.

[11] Kratz, H. R., Phys. Rev. 75 (1949) 1844.

[12] Nesmeyanov, A. N., Vapor pressure of the chemical elements (Elsevier Publishing Company Amsterdam, London, New York) 1963.

[13] Moore, C. E., Atomic Energy Levels III N.B.S. (1958).

[14] Chang, C. S. and Stehle, P., Phys. Rev. Lett. 30 (1973) 1283.

[15] Delone, G. A., Delone, N. B. and Piskova, G. K., Zh. Eksp. Teor. Fiz. 62 (1972) 1272 (Sov. Phys. JETP 35 (1972) 672).

[16] Voronov, G. S. and Delone, N. B., Sov. Phys. JETP 23 (1966) 54.

[17] Crance, M. and Feneuille, S., J. Physique Lett. 37 (1976) L-333. 